\title{
Transfer of Temporal Logic Formulas in Reinforcement Learning
}

\author{
Zhe Xu and Ufuk Topcu \\ University of Texas at Austin \\ \{zhexu, utopcu\}@utexas.edu
}

\begin{abstract}
Transferring high-level knowledge from a source task to a target task is an effective way to expedite reinforcement learning (RL). For example, propositional logic and first-order logic have been used as representations of such knowledge. We study the transfer of knowledge between tasks in which the timing of the events matters. We call such tasks temporal tasks. We concretize similarity between temporal tasks through a notion of logical transferability, and develop a transfer learning approach between different yet similar temporal tasks. We first propose an inference technique to extract metric interval temporal logic (MITL) formulas in sequential disjunctive normal form from labeled trajectories collected in RL of the two tasks. If logical transferability is identified through this inference, we construct a timed automaton for each sequential conjunctive subformula of the inferred MITL formulas from both tasks. We perform RL on the extended state which includes the locations and clock valuations of the timed automata for the source task. We then establish mappings between the corresponding components (clocks, locations, etc.) of the timed automata from the two tasks, and transfer the extended Q-functions based on the established mappings. Finally, we perform RL on the extended state for the target task, starting with the transferred extended Q-functions. Our implementation results show, depending on how similar the source task and the target task are, that the sampling efficiency for the target task can be improved by up to one order of magnitude by performing RL in the extended state space, and further improved by up to another order of magnitude using the transferred extended Q-functions.
\end{abstract}

\section{Introduction}

Reinforcement learning (RL) has been successful in numerous applications. In practice though, it often requires extensive exploration of the environment to achieve satisfactory performance, especially for complex tasks with sparse rewards [Wang and Taylor, 2017].
The sampling efficiency and performance of RL can be improved if some high-level knowledge can be incorporated in the learning process [Toro Icarte et al., 2018a]. Such knowledge can be also transferred from a source task to a target task if these tasks are logically similar [Taylor and Stone, 2007]. For example, propositional logic and first-order logic have been used as representations of knowledge in the form of $\log$ ical structures for transfer learning [Mihalkova et al., 2007]. They showed that incorporating such logical similarities can expedite RL for the target task [Torrey et al., 2008].

The transfer of high-level knowledge can be also applied to tasks where the timing of the events matters. We call such tasks as temporal tasks. Consider the gridworld example in Figure 1. In the source task, the robot should first reach a green region $G^{\mathrm{S}}$ and stay there for at least 4 time units, then reach another yellow region $Y^{\mathrm{S}}$ within 40 time units. In the target task, the robot should first reach a green region $G^{\mathrm{T}}$ and stay there for at least 5 time units, then reach another yellow region $Y^{\mathrm{T}}$ within 40 time units. In both tasks, the green and yellow regions are a priori unknown to the robot. After 40 time units, the robot obtains a reward of 100 if it has completed the task and obtains a reward of -10 otherwise. It is intuitive that the two tasks are similar at a high level despite the differences in the specific regions in the workspace and timing requirements.

Transfer learning between temporal tasks is complicated due to the following factors: (a) No formally defined criterion exists for logical similarities between temporal tasks. (b) Logical similarities are often implicit and need to be identified from data. (c) There is no known automated mechanism to transfer the knowledge based on logical similarities.

In this paper, we propose a transfer learning approach for temporal tasks in two levels: transfer of logical structures and

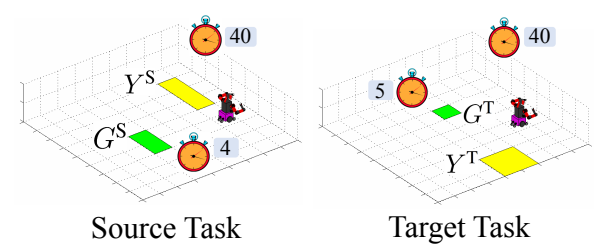

Figure 1: An illustrative example where the source task and the target task are logically similar. 
transfer of low-level implementations. For ease of presentation, we focus on Q-learning [Watkins and Dayan, 1992], while the general methodology applies readily to other forms of RL.

In the first level, we represent the high-level knowledge in temporal logic [Pnueli, 1977], which has been used in many applications in robotics and artificial intelligence [KressGazit et al., 2011; To et al., 2016]. Specifically, we use a fragment of metric interval temporal logic (MITL) with bounded time intervals. We transfer such knowledge from a source task to a target task based on the hypothesis of logical transferability (this notion will be formalized in Section 4.1 ) between the two tasks.

To identify logical transferability, we develop an inference technique that extracts informative MITL formulas (formalized in Section 3 ) in sequential disjunctive normal form. If the inference process indeed identifies logical transferability, we construct a timed automaton for each sequential conjunctive subformula of the inferred MITL formulas. We combine the locations and clock valuations of the timed automaton with the state of the robot to form an extended state, and perform RL in the extended state space for the target task.

In the second level, we transfer the extended $Q$-functions (i.e., Q-function on the extended states) from the source task to the target task if logical transferability is identified in the first level. We first perform RL in the extended state space for the source task. Next, we establish mappings between the corresponding components (clocks, locations, etc.) of the timed automata from the two tasks based on the identified logical transferability. Finally, we transfer the obtained optimal extended Q-functions from the source task to the target task based on these mappings, and perform RL for the target task starting with the transferred extended Q-functions.

The implementation of the proposed approach shows, in both levels, that the sampling efficiency is significantly improved for RL of the target task.

\subsection{Related Work}

Our work is closely related to the work on RL with temporal logic specifications [Aksaray et al., 2016; Li et al., 2017; Toro Icarte et al., 2018b; Fu and Topcu, 2014; Wen et al., 2017; Alshiekh et al., 2018]. The current results mostly rely on the assumption that the high-level knowledge (i.e., temporal logic specifications) are given, while in reality they are often implicit and need to be inferred from data.

The methods for inferring temporal logic formulas from data can be found in [Hoxha et al., 2017; Kong et al., 2017; Bombara et al., 2016; Neider and Gavran, 2018; Xu et al., 2018; Xu and Julius, 2018; Vazquez-Chanlatte et al., 2018; $\mathrm{Xu}$ et al., 2019; Shah et al., 2018]. The inference method used in this paper is inspired from [Bombara et al., 2016] and [Xu et al., 2019].

While there has been no existsing work on RL-based transfer learning utilizing similarity between temporal logic formulas, the related work on transferring first-order logical structures or rules for expediting RL can be found in [Taylor and Stone, 2007; Torrey and Shavlik, 2010; Torrey et al., 2008], and the related work on transferring logical relations for action-model acquisition can be found in [Zhuo and Yang, 2014].

\section{Preliminaries}

\subsection{Metric Interval Temporal Logic}

Let $\mathbb{B}=\{\top, \perp\}$ (tautology and contradiction, respectively) be the Boolean domain and $\mathbb{T}=\{0,1,2, \ldots\}$ be a discrete set of time indices. The underlying system is modeled by a Markov decision process (MDP) $\mathcal{M}=(S, A, P)$, where the state space $S$ and action set $A$ are finite, $P: S \times A \times$ $S \rightarrow[0,1]$ is a transition probability distribution. A trajectory $s_{0: L}=s_{0} s_{1} \cdots s_{L}$ describing an evolution of the MDP $\mathcal{M}$ is a function from $\mathbb{T}$ to $S$. Let $A P$ be a set of atomic predicates.

The syntax of the MITL $_{f}$ fragment of time-bounded MITL formulas is defined recursively as follows ${ }^{1}$ :

$$
\phi:=\top|\rho| \neg \phi\left|\phi_{1} \wedge \phi_{2}\right| \phi_{1} \vee \phi_{2}\left|\diamond_{I} \phi\right| \square_{I} \phi,
$$

where $\rho \in A P$ is an atomic predicate; $\neg$ (negation), $\wedge$ (conjunction), $\vee$ (disjunction) are Boolean connectives; $\diamond$ (eventually) and $\square$ (always) are temporal operators; and $I$ is a bounded interval of the form $I=\left[i_{1}, i_{2}\right]\left(i_{1}<i_{2}, i_{1}, i_{2} \in \mathbb{T}\right)$. For example, the MITL $_{f}$ formula $\square_{[2,5]}(x>3)$ reads as " $x$ is always greater than 3 during the time interval $[2,5]$ '.

A timed word generated by a trajectory $s_{0: L}$ is defined as a sequence $\left(\mathcal{L}\left(s_{t_{1}}\right), t_{1}\right), \ldots,\left(\mathcal{L}\left(s_{t_{m}}\right), t_{m}\right)$, where $\mathcal{L}: S \rightarrow$ $2^{\mathcal{A P}}$ is a labeling function assigning to each state $s \in S$ a subset of atomic predicates in $\mathcal{A P}$ that hold true at state $s$, $t_{1}=0, t_{m}=L, t_{k-1}<t_{k}(k \in[2, m])$ and for $k \in[1, m-$ 1], $t_{k+1}$ is the largest time index such that $\mathcal{L}\left(s_{t}\right)=\mathcal{L}\left(s_{t_{k}}\right)$ for all $t \in\left[t_{k}, t_{k+1}\right)$. The satisfaction of an $\mathrm{MITL}_{f}$ formula by timed words as Boolean semantics can be found in [Alur et al., 1996]. We say that a trajectory $s_{0: L}$ satisfies an $\mathrm{MITL}_{f}$ formula $\phi$, denoted as $s_{0: L} \models \phi$, if and only if the timed word generated by $s_{0: L}$ satisfies $\phi$. As the time intervals $I$ in MITL $_{f}$ formulas are bounded intervals, MITL $f$ formulas can be satisfied and violated by trajectories of finite lengths.

\subsection{Timed Automaton}

Let $C$ be a finite set of clock variables. The set $\mathcal{C}_{C}$ of clock constraints is defined by [Ouaknine and Worrell, 2005]

$$
\varphi_{C}:=\top|c \bowtie k| \varphi_{1} \wedge \varphi_{2},
$$

where $k \in \mathbb{N}, c \in C$ and $\bowtie \in\{<, \leq,>, \geq\}$.

Definition 1. [Alur and Dill, 1994] A timed automaton is a tuple $\mathcal{A}=\left(\Sigma, \mathcal{Q}, q^{0}, C, \mathcal{F}, \Delta\right)$, where $\Sigma$ is a finite alphabet of input symbols, $\mathcal{Q}$ is a set of locations, $q^{0} \in \mathcal{Q}$ is the initial location, $C$ is a finite set of clocks, $\mathcal{F} \subset \mathcal{Q}$ is a set of accepting locations, $\Delta \subset \mathcal{Q} \times \Sigma \times \mathcal{Q} \times \mathcal{C}_{C} \times 2^{C}$ is the transition function, $e=\left(q, \sigma, q^{\prime}, \varphi_{C}, r_{C}\right) \in \Delta$ represents a transition from $q$ to $q^{\prime}$ labeled by $\sigma$, provided the precondition $\varphi_{C}$ on the clocks is met, $r_{C}$ is the set of clocks that are reset to zero.

Remark 1. We focus on timed automata with discrete time, which are also called tick automata in [Gruber et al., 2005].

\footnotetext{
${ }^{1}$ Although other temporal operators such as "Until " $(\mathcal{U})$ may also appear in the full syntax of MITL, they are omitted from the syntax here as they can be hard to interpret and are not often used for the inference of temporal logic formulas [Kong et al., 2017].
} 
A timed automaton $\mathcal{A}$ is deterministic if and only if for each location and input symbol there is at most one transition to the next location. We denote by $v=\left(v_{1}, \ldots, v_{|C|}\right) \in V \subset$ $\mathbb{T}^{|C|}$ the clock valuation of $\mathcal{A}$ (we denote by $|C|$ the cardinality of $C$ ), where $v_{k} \in V_{k} \subset \mathbb{T}$ is the value of clock $c_{k} \in C$. For a timed word $\nu=\left(\sigma_{0}, t_{0}\right),\left(\sigma_{1}, t_{1}\right), \ldots,\left(\sigma_{m}, t_{m}\right)$ (where $t_{0}<t_{1}<\cdots<t_{m}, \sigma_{k} \in \Sigma$ for $\left.k \in[0, m]\right)$ and writing $d_{k}:=t_{k+1}-t_{k}$, a run of $\mathcal{A}$ on $\nu$ is defined as

$\left(q^{0}, v^{0}\right) \stackrel{\sigma_{0}}{\longrightarrow}\left(q^{1}, v^{1}\right) \stackrel{d_{0}}{\rightarrow}\left(q^{2}, v^{2}\right) \stackrel{\sigma_{1}}{\longrightarrow}\left(q^{3}, v^{3}\right) \stackrel{d_{1}}{\longrightarrow} \ldots \stackrel{d_{m-1}}{\longrightarrow}$ $\left(q^{2 m}, v^{2 m}\right) \stackrel{\sigma_{m}}{\longrightarrow}\left(q^{2 m+1}, v^{2 m+1}\right)$,

where the flow-step relation is defined by $(q, v) \stackrel{d}{\rightarrow}(q, v+d)$ where $d \in \mathbb{R}_{>0}$; the edge-step relation is defined by $(q, v) \stackrel{\sigma}{\rightarrow}$ $\left(q^{\prime}, v^{\prime}\right)$ if and only if there is an edge $\left(q, \sigma, q^{\prime}, \varphi_{C}, r_{C}\right) \in \Delta$ such that $\sigma \in \Sigma, v$ satisfies $\varphi_{C}, v_{k}^{\prime}=0$ for all $c_{k} \in r_{C}$ and $v_{k}^{\prime}=v_{k}$ for all $c_{k} \notin r_{C}$. A finite run is accepting if the last location in the run belongs to $\mathcal{F}$. A timed word $\nu$ is accepted by $\mathcal{A}$ if there is some accepting run of $\mathcal{A}$ on $\nu$.

\section{Information-Guided Inference of Temporal Logic Formulas}

We now introduce the information gain provided by an $\mathrm{MITL}_{f}$ formula, the problem formulation and the algorithm to extract $\mathrm{MITL}_{f}$ formulas from labeled trajectories.

\subsection{Information Gain of MITL $_{f}$ Formulas}

We denote by $\mathcal{B}_{L}$ the set of all possible trajectories with length $L$ generated by the $\operatorname{MDP} \mathcal{M}$, and use $\mathcal{G}_{L}: \mathcal{B}_{L} \rightarrow[0,1]$ to denote a prior probability distribution (e.g., uniform distribution) over $\mathcal{B}_{L}$. We use $\mathbb{P}_{\mathcal{B}_{L}, \phi}$ to denote the probability of a trajectory $s_{0: L}$ satisfying $\phi$ in $\mathcal{B}_{L}$ based on $\mathcal{G}_{L}$.

Definition 2. Given a prior probability distribution $\mathcal{G}_{L}$ and an MITL $_{f}$ formula $\phi$ such that $\mathbb{P}_{\mathcal{B}_{L}, \phi}>0$, we define $\overline{\mathcal{G}}_{L}^{\phi}$ : $\mathcal{B}_{L} \rightarrow[0,1]$ as the posterior probability distribution, given that $\phi$ evaluates to true, which is expressed as

$$
\overline{\mathcal{G}}_{L}^{\phi}\left(s_{0: L}\right):=\left\{\begin{array}{lc}
\frac{\mathcal{G}_{L}\left(s_{0: L}\right)}{\mathbb{P}_{\mathcal{B}_{L}, \phi}}, & \text { if } s_{0: L} \models \phi \\
0, & \text { otherwise }
\end{array}\right.
$$

The expression of $\overline{\mathcal{G}}_{L}^{\phi}$ can be derived using Bayes' theorem. We use the fact that the probability of $\phi$ evaluating to true given $s_{0: L}$ is 1 , if $s_{0: L}$ satisfies $\phi$; and it is 0 otherwise.

Definition 3. When the prior probability distribution $\mathcal{G}_{L}$ is updated to the posterior probability distribution $\overline{\mathcal{G}}_{L}^{\phi}$, we define the information gain as

$$
\mathcal{I}\left(\mathcal{G}_{L}, \overline{\mathcal{G}}_{L}^{\phi}\right):=D_{\mathrm{KL}}\left(\overline{\mathcal{G}}_{L}^{\phi} \| \mathcal{G}_{L}\right) / L
$$

where $D_{\mathrm{KL}}\left(\overline{\mathcal{G}}_{L}^{\phi} \| \mathcal{G}_{L}\right)$ is the Kullback-Leibler divergence from $\mathcal{G}_{L}$ to $\overline{\mathcal{G}}_{L}^{\phi}$.

Proposition 1. For an $M I T L_{f}$ formula $\phi$, if $\mathbb{P}_{\mathcal{B}_{L}, \phi}>0$, then

$$
\mathcal{I}\left(\mathcal{G}_{L}, \overline{\mathcal{G}}_{L}^{\phi}\right)=-\log \mathbb{P}_{\mathcal{B}_{L}, \phi} / L
$$

Proof. Straightforward from Definitions 2 and 3.
If $\phi=\top$, then $\mathbb{P}_{\mathcal{B}_{L}, \phi}=1$ and $\mathcal{I}\left(\mathcal{G}_{L}, \overline{\mathcal{G}}_{L}^{\phi}\right)=0$, i.e., tautologies provide no information gain. For completeness, we also define that the information gain $\mathcal{I}\left(\mathcal{G}_{L}, \overline{\mathcal{G}}_{L}^{\phi}\right)=0$ if $\mathbb{P}_{\mathcal{B}_{L}, \phi}=0$. So if $\phi=\perp$, then $\mathbb{P}_{\mathcal{B}_{L}, \phi}=0$ and $\mathcal{I}\left(\mathcal{G}_{L}, \overline{\mathcal{G}}_{L}^{\phi}\right)=0$, i.e., contradictions provide no information gain.

For two MITL formulas $\phi_{1}$ and $\phi_{2}$, we say $\phi_{1}$ is more informative than $\phi_{2}$ with respect to the prior probability distribution $\mathcal{G}_{L}$ if $\mathcal{I}\left(\mathcal{G}_{L}, \overline{\mathcal{G}}_{L}^{\phi_{1}}\right)>\mathcal{I}\left(\mathcal{G}_{L}, \overline{\mathcal{G}}_{L}^{\phi_{2}}\right)$

Based on Proposition 1, the computation of the information gain requires the computation of $\mathbb{P}_{\mathcal{B}_{L}, \phi}$. We point the reader to [Xu et al., 2019] for a recursive method to compute $\mathbb{P}_{\mathcal{B}_{L}, \phi}$.

\subsection{Problem Formulation}

We now provide some related definitions for formulating the inference problem. Let a set $\mathcal{P}$ of primitive structures [Bombara et al., 2016] used in the rest of the paper be

$$
\mathcal{P}:=\left\{\diamond_{I} \rho, \square_{I} \rho, \diamond_{I} \square_{I^{\prime}} \rho, \square_{I} \diamond_{I^{\prime}} \rho\right\},
$$

where $I=\left[i_{1}, i_{2}\right]\left(i_{1}<i_{2}, i_{1}, i_{2} \in \mathbb{T}\right), I^{\prime}=\left[0, i_{2}\right]\left(i_{2}>0\right.$, $\left.i_{2} \in \mathbb{T}\right)$, and $\rho$ is an atomic predicate. We call an $\operatorname{MITL}_{f}$ formula $\phi$ a primitive $M I T L_{f}$ formula if $\phi$ follows one of the primitive structures in $\mathcal{P}$ or the negation of such a structure.

Definition 4. For an $M I T L_{f}$ formula $\phi$, we define the starteffect time $t_{\mathrm{S}}(\phi)$ and end-effect time $t_{\mathrm{e}}(\phi)$ recursively as

$$
\begin{aligned}
t_{\mathrm{s}}(\rho) & =t_{\mathrm{e}}(\rho)=0, t_{\mathrm{s}}(\neg \phi)=t_{\mathrm{s}}(\phi), t_{\mathrm{e}}(\neg \phi)=t_{\mathrm{e}}(\phi), \\
t_{\mathrm{s}}\left(\phi_{1} \wedge \phi_{2}\right) & =\min \left\{t_{\mathrm{s}}\left(\phi_{1}\right), t_{\mathrm{s}}\left(\phi_{2}\right)\right\}, \\
t_{\mathrm{e}}\left(\phi_{1} \wedge \phi_{2}\right) & =\max \left\{t_{\mathrm{e}}\left(\phi_{1}\right), t_{\mathrm{e}}\left(\phi_{2}\right)\right\}, \\
t_{\mathrm{s}}\left(\diamond_{\left[t_{1}, t_{2}\right]} \phi\right) & =t_{\mathrm{s}}(\phi)+t_{1}, \quad t_{\mathrm{e}}\left(\diamond_{\left[t_{1}, t_{2}\right]} \phi\right)=t_{\mathrm{e}}(\phi)+t_{2}, \\
t_{\mathrm{s}}\left(\square_{\left[t_{1}, t_{2}\right]} \phi\right) & =t_{\mathrm{s}}(\phi)+t_{1}, \quad t_{\mathrm{e}}\left(\square_{\left[t_{1}, t_{2}\right]} \phi\right)=t_{\mathrm{e}}(\phi)+t_{2} .
\end{aligned}
$$

Definition 5. An MITL $L_{f}$ formula $\phi$ is in disjunctive normal form if $\phi$ is expressed in the form of $\left(\phi_{1}^{1} \wedge \cdots \wedge \phi_{1}^{n_{1}}\right) \vee \cdots \vee$ $\left(\phi_{m}^{1} \wedge \cdots \wedge \phi_{m}^{n_{m}}\right)$, where each $\phi_{i}^{j}$ is a primitive $M I T L_{f}$ formula (also called primitive subformula of $\phi$ ). If, for any $i \in[1, m]$ and for all $j, k \in\left[1, n_{i}\right]$ such that $j<k$, it holds that $t_{\mathrm{e}}\left(\phi_{i}^{j}\right)<t_{\mathrm{s}}\left(\phi_{i}^{k}\right)$, then we say $\phi$ is in sequential disjunctive normal form (SDNF) and we call each $\phi_{i}:=\phi_{i}^{1} \wedge \cdots \wedge \phi_{i}^{n_{i}}$ a sequential conjunctive subformula.

In the following, we consider $\mathrm{MITL}_{f}$ formulas only in the SDNF for reasons that will become clear in Section 4 . We define the size of an $\mathrm{MITL}_{f}$ formula $\phi$ in the SDNF, denoted as $\varrho(\phi)$, as the number of primitive $\mathrm{MITL}_{f}$ formulas in $\phi$.

Suppose that we are given a set $\mathcal{S}_{L}=\left\{\left(s_{0: L}^{k}, l_{k}\right)\right\}_{k=1}^{N_{\mathcal{S}_{L}}}$ of labeled trajectories, where $l_{k}=1$ and $l_{k}=-1$ represent desired and undesired behaviors, respectively. We define the satisfaction signature $g_{\phi}\left(s_{0: L}^{k}\right)$ of a trajectory $s_{0: L}^{k}$ as follows: $g_{\phi}\left(s_{0: L}^{k}\right)=1$, if $s_{0: L}^{k}$ satisfies $\phi$; and $g_{\phi}\left(s_{0: L}^{k}\right)=-1$, if $s_{0: L}^{k}$ does not satisfy $\phi$. Note that here we assume that $L$ is sufficiently large, thus $s_{0: L}^{k}$ either satisfies or violates $\phi$. A labeled trajectory $\left(s_{0: L}^{k}, l_{k}\right)$ is misclassified by $\phi$ if $g_{\phi}\left(s_{0: L}^{k}\right) \neq l_{k}$. We use $C R\left(S_{L}, \phi\right)=\left|\left\{\left(s_{0: L}^{k}, l_{k}\right) \in \mathcal{S}_{L}: g_{\phi}\left(s_{0: L}^{k}\right)=l_{k}\right\}\right| /\left|\mathcal{S}_{L}\right|$ to denote the classification rate of $\phi$ in $\mathcal{S}_{L}$.

Problem 1. Given a set $\mathcal{S}_{L}=\left\{\left(s_{0: L}^{k}, l_{k}\right)\right\}_{k=1}^{N_{\mathcal{S}_{L}}}$ of labeled trajectories, a prior probability distribution $\overline{\mathcal{G}}_{L}$, real constant $\zeta \in(0,1]$ and integer constant $\varrho_{\mathrm{th}} \in(0, \infty)$, construct 
an MITL $_{f}$ formula $\phi$ in the SDNF that maximizes $\mathcal{I}\left(\mathcal{G}_{L}, \overline{\mathcal{G}}_{L}^{\phi}\right)$ while satisfying

- the classification constraint $C R\left(\mathcal{S}_{L}, \phi\right) \geq \zeta$ and

- the size constraint $\varrho(\phi) \leq \varrho_{\text {th }}$.

Intuitively, as there could be many $\mathrm{MITL}_{f}$ formulas that satisfy the classification constraint and the size constraint, we intend to obtain the most informative one to be utilized and transferred as features of desired behaviors.

We call an $\mathrm{MITL}_{f}$ formula that satisfies both the two constraints of Problem 1 a satisfying formula for $\mathcal{S}_{L}$.

\subsection{Solution Based on Decision Tree}

We propose an inference technique, which is inspired by [Bombara et al., 2016] and [Xu et al., 2019], in order to solve Problem 1. The technique consists of two steps. In the first step, we construct a decision tree where each non-leaf node is associated with a primitive $\mathrm{MITL}_{f}$ formula (see the formulas inside the circles in Figure 2). In the second step, we convert the constructed decision tree to an $\mathrm{MITL}_{f}$ formula in the SDNF.

In Algorithm 1, we construct the decision tree by recursively calling the MITLtree procedure from the root node to each leaf node. There are three inputs to the MITLtree procedure: (1) a set $\mathcal{S}$ of labeled trajectories assigned to the current node; (2) a formula $\phi^{\text {path }}$ to reach the current node (also called the path formula, see the formulas inside the rectangles in Figure 2); and (3) the depth $h$ of the current node. The set $\mathcal{S}$ assigned to the root node is initialized as $\mathcal{S}_{L}$ in Problem 1, $\phi^{\text {path }}$ and $h$ are initialized as $\top$ and 0 , respectively.

For each node, we set a criterion $\operatorname{stop}\left(\phi^{\text {path }}, h, \mathcal{S}\right)$ to determine whether it is a leaf node (Line 2). Each leaf node is associated with label 1 or -1 , depending on whether more than $50 \%$ of the labeled trajectories assigned to that node are with label 1 or not.

At each non-leaf node $z$, we construct a primitive MITL $_{f}$ formula $\phi_{\theta}$ parameterized by $\theta \in \Upsilon_{z}$, where

$$
\begin{aligned}
\Upsilon_{z}:= & \left\{\theta \mid\left[t_{\mathrm{s}}\left(\phi_{\theta}\right), t_{\mathrm{e}}\left(\phi_{\theta}\right)\right] \cap\left[t_{\mathrm{s}}\left(\phi^{\prime}\right), t_{\mathrm{e}}\left(\phi^{\prime}\right)\right]=\emptyset\right. \\
& \text { for each primitive subformula } \left.\phi^{\prime} \text { of } \phi^{\mathrm{path}}\right\} .
\end{aligned}
$$

For example, for an $\operatorname{MITL}_{f}$ formula $\phi_{\theta}=\square_{\left[i_{1}, i_{2}\right]}(x>a)$, we have $\theta=\left[i_{1}, i_{2}, a\right]$. If $\phi^{\text {path }}=\diamond_{[1,15]} \square_{[0,4]}(x>3)$, then $\Upsilon_{z}$ ensures that the start-effect time of $\phi_{\theta}$ is later than the endeffect time of $\phi^{\text {path }}$ (which is 19). Essentially $\Upsilon_{z}$ guarantees that the primitive $\mathrm{MITL}_{f}$ formula $\phi_{\theta}$ and primitive subformulas of $\phi^{\text {path }}$ can be reordered to form a sequential conjunctive subformula (see Definition 5).

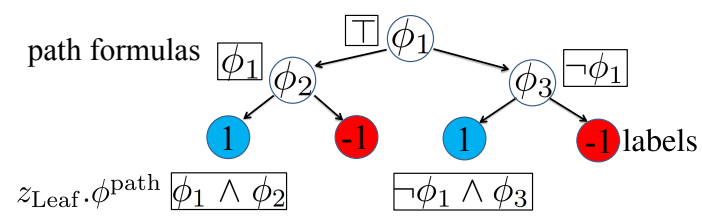

Figure 2: Illustration of a decision tree which can be converted to an MITL $_{f}$ formula in the SDNF.

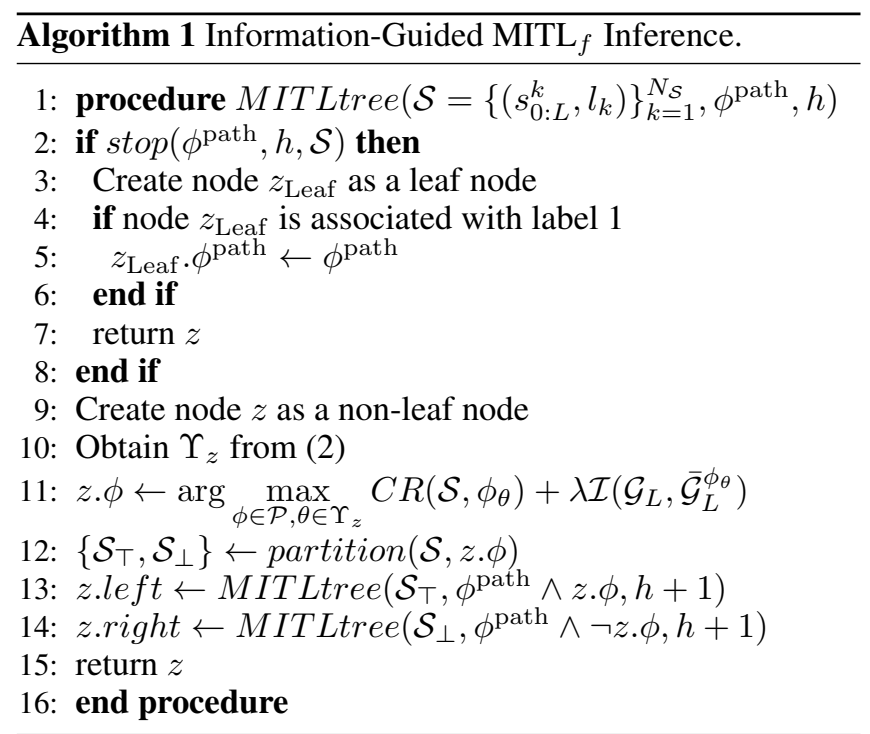

We use particle swarm optimization (PSO) [Eberhart and Shi, 2001] to optimize $\theta$ for each primitive structure from $\mathcal{P}$ and compute a primitive MITL $_{f}$ formula $z . \phi=\phi_{\theta}^{*}$ which maximizes the objective function

$$
J\left(\mathcal{S}, \phi_{\theta}\right):=C R\left(\mathcal{S}, \phi_{\theta}\right)+\lambda \mathcal{I}\left(\mathcal{G}_{L}, \overline{\mathcal{G}}_{L}^{\phi_{\theta}}\right)
$$

in Line 11, where $\lambda$ is a weighting factor.

With $z . \phi$, we partition the set $\mathcal{S}$ into $\mathcal{S}_{\top}$ and $\mathcal{S}_{\perp}$, where the trajectories in $\mathcal{S}_{\top}$ and $\mathcal{S}_{\perp}$ satisfy and violate $z . \phi$, respectively (Line 12). Then the procedure is called recursively to construct the left and right sub-trees for $\mathcal{S}_{\top}$ and $\mathcal{S}_{\perp}$, respectively (Lines 13, 14).

After the decision tree is constructed, for each leaf node associated with label 1 , it is also associated with a path formula $z_{\text {Leaf }} \phi^{\text {path }}$ (Line 4 to Line 6 ). The path formula $z_{\text {Leaf }} . \phi^{\text {path }}$ is constructed recursively from the associated primitive $\mathrm{MITL}_{f}$ formulas along the path from the root node to the parent of the leaf node (see Figure 2). We rearrange the primitive subformulas of each $z_{\text {Leaf }} . \phi^{\text {path }}$ in the order of increasing start-effect time to obtain a sequential conjunctive subformula. We then connect all the obtained sequential conjunctive subformulas with disjunctions. In this way, the obtained decision tree can be converted to an $\mathrm{MITL}_{f}$ formula in the SDNF. As in the example shown in Figure 2, if $t_{\mathrm{S}}\left(\phi_{1}\right)<t_{\mathrm{S}}\left(\phi_{2}\right)$ and $t_{\mathrm{s}}\left(\neg \phi_{1}\right)<t_{\mathrm{s}}\left(\phi_{3}\right)$, then the decision tree can be converted to $\left(\phi_{1} \wedge \phi_{2}\right) \vee\left(\neg \phi_{1} \wedge \phi_{3}\right)$ in the SDNF. If $t_{\mathrm{S}}\left(\phi_{2}\right)<t_{\mathrm{s}}\left(\phi_{1}\right)$ and $t_{\mathrm{s}}\left(\phi_{3}\right)<t_{\mathrm{s}}\left(\neg \phi_{1}\right)$, then the decision tree can be converted to $\left(\phi_{2} \wedge \phi_{1}\right) \vee\left(\phi_{3} \wedge \neg \phi_{1}\right)$ in the SDNF.

We set the criterion stop $\left(\phi^{\text {path }}, h, \mathcal{S}\right)$ as follows. If at least $\zeta$ (e.g., 95\%) of the labeled trajectories assigned to the node are with the same label (Condition I) or the depth $h$ of the node reaches a set maximal depth $h_{\max }$ (Condition II) or $\Upsilon_{z}$, as defined in (2), becomes the empty set (Condition III), then the node is a leaf node. If condition I holds for each leaf mode, then the obtained MITL $_{f}$ formula satisfies the classification constraint of Problem 1. If we set $h_{\max } 2^{h_{\max }-1} \leq \varrho_{\mathrm{th}}$, then the size constraint is guaranteed to be satisfied.

The complexity of Algorithm 1 for the average case can be 
determined through the Akra-Bazzi method as follows [Bombara et al., 2016]:

$$
\Theta\left(N_{\mathcal{S}} \cdot\left(1+\int_{1}^{N_{\mathcal{S}}} \frac{f(u)}{u^{2}} \mathrm{~d} u\right)\right),
$$

where $f\left(N_{\mathcal{S}}\right)$ is the complexity of the local PSO algorithm for $N_{\mathcal{S}}$ labeled trajectories, and $\Theta(\cdot)$ denotes the two-sided asymptotic notation for complexity bound.

\section{Transfer Learning of Temporal Tasks Based on Logical Transferability}

In this section, we first introduce the notion of logical transferability. Then, we present the framework and algorithms for utilizing logical transferability for transfer learning.

\subsection{Logical Transferability}

To define logical transferability, we first define the structural transferability between two $\mathrm{MITL}_{f}$ formulas.

For each primitive $\mathrm{MITL}_{f}$ formula $\phi$, we use $O_{T}(\phi)$ to denote the temporal operator in $\phi$. For example, $O_{T}\left(\diamond_{[5,8]}(x>\right.$ $3))=\diamond$ (eventually) and $O_{T}\left(\square_{[0,8]} \diamond_{[0,4]}(x<5)\right)=\square \diamond$ (always eventually).

Definition 6. $T$ wo $M I T L_{f}$ formulas (in the SDNF)

$$
\phi=\left(\phi_{1}^{1} \wedge \cdots \wedge \phi_{1}^{n_{1}}\right) \vee \cdots \vee\left(\phi_{m}^{1} \wedge \cdots \wedge \phi_{m}^{n_{m}}\right)
$$

and

$$
\hat{\phi}=\left(\hat{\phi}_{1}^{1} \wedge \cdots \wedge \hat{\phi}_{1}^{\hat{n}_{1}}\right) \vee \cdots \vee\left(\hat{\phi}_{\hat{m}}^{1} \wedge \cdots \wedge \hat{\phi}_{\hat{m}}^{\hat{n}_{\hat{m}}}\right)
$$

are structurally equivalent, if and only if the followings hold: (1) $m=\hat{m}$ and, for every $i \in[1, m], n_{i}=\hat{n}_{i}$; and

(2) For every $i \in[1, m]$ and every $j \in\left[1, n_{i}\right], O_{T}\left(\phi_{i}^{j}\right)=$ $O_{T}\left(\hat{\phi}_{i}^{j}\right)$.

Definition 7. For two $M I T L_{f}$ formulas $\phi_{1}$ and $\phi_{2}$ in the SDNF, $\phi_{2}$ is structurally transferable from $\phi_{1}$ if and only if either of the following conditions holds:

1) $\phi_{1}$ and $\phi_{2}$ are structurally equivalent;

2) $\phi_{2}$ is in the form of $\phi_{2}^{1} \vee \cdots \vee \phi_{2}^{p}(p>1)$, where each $\phi_{2}^{k}(k=1, \ldots, p)$ is structurally equivalent with $\phi_{1}$.

Suppose that we are given a source task $\mathcal{T}^{\mathrm{S}}$ in the source environment $\mathcal{E}^{\mathrm{S}}$ and a target task $\mathcal{T}^{\mathrm{T}}$ in the target environment $\mathcal{E}^{\mathrm{T}}$, with two sets $\mathcal{S}_{L}^{\mathrm{S}}$ and $\mathcal{S}_{L}^{\mathrm{T}}$ of labeled trajectories collected during the initial episodes of RL (which we call the data collection phase) in $\mathcal{E}^{\mathrm{S}}$ and $\mathcal{E}^{\mathrm{T}}$ respectively. The trajectories are labeled based on a given task-related performance criterion. To ensure the quality of inference, the data collection phase is chosen such that both $\mathcal{S}_{L}^{\mathrm{S}}$ and $\mathcal{S}_{L}^{\mathrm{T}}$ contain sufficient labeled trajectories with both label 1 and label -1.We give the following definition for logical transferability.

Definition 8. $\mathcal{T}^{T}$ is logically transferable from $\mathcal{T}^{S}$ based on $\mathcal{S}_{L}^{S}, \mathcal{S}_{L}^{T}, \zeta$ and $\varrho_{\text {th }}$ (as defined in Problem 1), if and only if there exist satisfying formulas $\phi^{S}$ for $\mathcal{S}_{L}^{S}$ and $\phi^{T}$ for $\mathcal{S}_{L}^{T}$ such that $\phi^{T}$ is structurally transferable from $\phi^{S}$.

In the following, we explain the proposed transfer learning approach based on logical transferability in two different levels. We provide a workflow diagram as an overview of the proposed transfer learning approach, as shown in Figure 3.

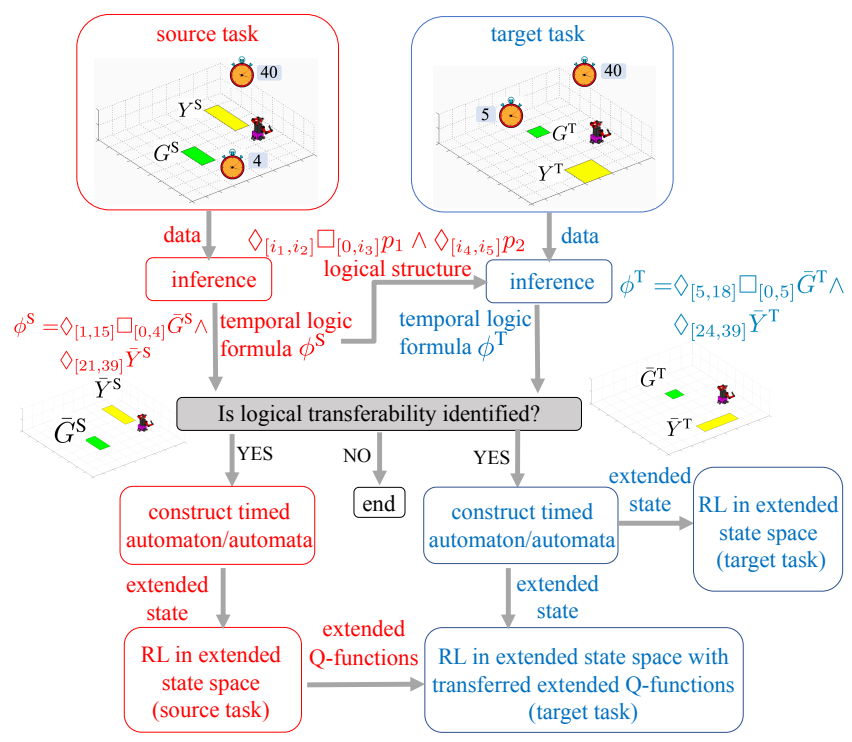

Figure 3: Workflow diagram of the proposed transfer learning approach based on logical transferability.

\subsection{Transfer of Logical Structures Based on Hypothesis of Logical Transferability}

We first introduce the transfer of logical structures between temporal tasks. To this end, we pose the hypothesis that the target task is logically transferable from the source task. If logical transferability can be indeed identified, we perform RL for the target task utilizing the transferred logical structure. Specifically, we take the following three steps:

Step 1: Extracting MITL $_{f}$ Formulas in the Source Task From $\mathcal{S}_{L}^{\mathrm{S}}$, we infer an $\mathrm{MITL}_{f}$ formula $\phi^{\mathrm{S}}$ using Algorithm 1. If $\phi^{\mathrm{S}}$ is a satisfying formula for $\mathcal{S}_{L}^{\mathrm{S}}$, we proceed to Step 2.

As in the introductory example, we obtain the satisfying formula $\phi^{\mathrm{S}}=\diamond_{[1,15]} \square_{[0,4]} \bar{G}^{\mathrm{S}} \wedge \diamond_{[21,39]} \bar{Y}^{\mathrm{S}}$ for $\mathcal{S}_{L}^{\mathrm{S}}$ (see Section 5 for details).

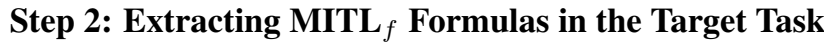

From $\mathcal{S}_{L}^{\mathrm{T}}$, we check if it is possible to infer a satisfying $\mathrm{MITL}_{f}$ formula $\phi^{\mathrm{T}}$ for $\mathcal{S}_{L}^{\mathrm{T}}$ such that $\phi^{\mathrm{T}}$ is structurally transferable from the inferred $\mathrm{MITL}_{f}$ formula $\phi^{\mathrm{S}}$ from the source task. We start from inferring an $\mathrm{MITL}_{f}$ formula that is structurally equivalent with $\phi^{\mathrm{S}}$. This can be done by fixing the temporal operators (the same with those of $\phi^{\mathrm{S}}$ ), then optimizing the parameters that appear in $\phi^{\mathrm{T}}$ (through PSO) for maximizing the objective function in (3). If a satisfying $\mathrm{MITL}_{f}$ formula is not found, we infer a MITL formula $\phi^{\mathrm{T}}$ in the form of $\phi^{\mathrm{T}}=\phi_{1}^{\mathrm{T}} \vee \phi_{2}^{\mathrm{T}}$, where $\phi_{1}^{\mathrm{T}}$ and $\phi_{2}^{\mathrm{T}}$ are both structurally equivalent with $\phi^{\mathrm{S}}$. In this way, we keep increasing the number of structurally equivalent formulas connected with disjunctions until a satisfying $\mathrm{MITL}_{f}$ formula is found, or the size constraint is violated (i.e., $\left.\varrho\left(\phi^{\mathrm{T}}\right)>\varrho_{\text {th }}\right)$. If a satisfying $\mathrm{MITL}_{f}$ formula is found, we proceed to Step 3; otherwise, logical transferability is not identified. 
As in the introductory example, we obtain the satisfying formula $\phi^{\mathrm{T}}=\diamond_{[5,18]} \square_{[0,5]} \bar{G}^{\mathrm{T}} \wedge \diamond_{[24,39]} \bar{Y}^{\mathrm{T}}$ for $\mathcal{S}_{L}^{\mathrm{T}}$ and $\phi^{\mathrm{T}}$ is structurally equivalent with $\phi^{\mathrm{S}}$, hence logical transferability is identified.

\section{Step 3: Constructing Timed Automata and Performing RL in the Extended State Space for the Target Task}

For the satisfying formula $\phi^{\mathrm{T}}=\phi_{1}^{\mathrm{T}} \vee \cdots \vee \phi_{m}^{\mathrm{T}}$ in the SDNF, we can construct a deterministic timed automaton (DTA) $\mathcal{A}^{\phi_{i}^{\mathrm{T}}}=\left\{2^{A P}, \mathcal{Q}^{\phi_{i}^{\mathrm{T}}}, q^{0 \phi_{i}^{\mathrm{T}}}, C^{\phi_{i}^{\mathrm{T}}}, \mathcal{F}^{\phi_{i}^{\mathrm{T}}}, \Delta \phi_{i}^{\mathrm{T}}\right\}$ [Alur et al., 1996] that accepts precisely the timed words that satisfy each sequential conjunctive subformula $\phi_{i}^{\mathrm{T}}$.

We perform RL in the extended state space $X^{\mathrm{T}}=\bigcup_{i} X_{i}^{\mathrm{T}}$, where each $X_{i}^{\mathrm{T}}=S^{\mathrm{T}} \times \mathcal{Q}^{\phi_{i}^{\mathrm{T}}} \times V^{\phi_{i}^{\mathrm{T}}}\left(S^{\mathrm{T}}\right.$ is the state space for the target task, $V^{\phi_{i}^{\mathrm{T}}}$ is the set of clock valuations for the clocks in $C^{\phi_{i}^{\mathrm{T}}}$ ) is a finite set of extended states. For each episode, the index $i$ is first selected based on some heuristic criterion. For example, if the atomic predicates correspond to the regions to be reached in the state space, we select $i$ such that the centroid of the region corresponding to the atomic predicate in $\phi_{i}^{1, \mathrm{~T}}$ (as in $\phi_{i}^{\mathrm{T}}=\phi_{i}^{1, \mathrm{~T}} \wedge \cdots \wedge \phi_{i}^{n_{i}, \mathrm{~T}}$ ) has the nearest (Euclidean) distance from the initial state $s_{0}^{\mathrm{T}}$. Then we perform $\mathrm{RL}$ in $X_{i}^{\mathrm{T}}$. For Q-learning, after taking action $a^{\mathrm{T}}$ at the current extended state $\chi_{i}^{\mathrm{T}}=\left(s^{\mathrm{T}}, q^{\mathrm{T}}, v^{\mathrm{T}}\right) \in X_{i}^{\mathrm{T}}$, a new extended state $\chi_{i}^{\prime \mathrm{T}}=\left(s^{\prime \mathrm{T}}, q^{\prime} \mathrm{T}, v^{\prime} \mathrm{T}\right) \in X_{i}^{\mathrm{T}}$ and a reward $R^{\mathrm{T}}$ are obtained. We have the following update rule for the extended $Q$-function values (denoted as $\bar{Q}$ ):

$\bar{Q}\left(\chi_{i}^{\mathrm{T}}, a^{\mathrm{T}}\right) \leftarrow(1-\alpha) \bar{Q}\left(\chi_{i}^{\mathrm{T}}, a^{\mathrm{T}}\right)+\alpha\left(R^{\mathrm{T}}+\gamma \max _{a^{\prime \mathrm{T}}} \bar{Q}\left(\chi_{i}^{\prime \mathrm{T}}, a^{\prime \mathrm{T}}\right)\right)$,

where $\alpha$ and $\gamma$ are the learning rate and discount factor, respectively.

As in the introductory example, we construct a DTA [see Figure 4 (b)] that accepts precisely the timed words that satisfy $\phi^{\mathrm{T}}$ as there is only one sequential conjunctive subformula in $\phi^{\mathrm{T}}$. We then perform RL in the extended state space $X^{\mathrm{T}}=S^{\mathrm{T}} \times \mathcal{Q}^{\phi^{\mathrm{T}}} \times V^{\phi^{\mathrm{T}}}$, where $S^{\mathrm{T}}$ is the state space in the $9 \times 9$ gridworld, $\mathcal{Q}^{\phi^{\mathrm{T}}}=\left\{q_{0}^{\mathrm{T}}, q_{1}^{\mathrm{T}}, q_{2}^{\mathrm{T}}, q_{3}^{\mathrm{T}}\right\}\left(q_{0}^{\mathrm{T}}=q^{0 \phi^{\mathrm{T}}}\right)$ and $V^{\phi^{\mathrm{T}}}=\{0,1, \ldots, 40\} \times\{0,1, \ldots, 40\}$ (the set of clock valuations for the clocks $c_{1}^{\mathrm{T}}$ and $c_{2}^{\mathrm{T}}$ ).

\subsection{Transfer of Extended Q-functions Based on Identified Logical Transferability}

Next, we introduce the transfer of extended Q-functions if logical transferability can be identified from Section 4.2.

We assume that the sets of actions in the source task and the target task are the same, denoted as $A$. For the satisfying formula $\phi^{\mathrm{S}}=\phi_{1}^{\mathrm{S}} \vee \cdots \vee \phi_{m}^{\mathrm{S}}$ in the SDNF, we construct a DTA corresponding to each $\phi_{i}^{\mathrm{S}}$ and perform Q-learning in the extended state space for the source task. We denote the obtained optimal extended Q-functions as $\bar{Q}^{\mathrm{S} *}\left(s^{\mathrm{S}}, q^{\mathrm{S}}, v^{\mathrm{S}}, a\right)$. In the following, we explain the details for transferring $\bar{Q}^{\mathrm{S} *}\left(s^{\mathrm{S}}, q^{\mathrm{S}}, v^{\mathrm{S}}, a\right)$ to the target task based on the identified logical transferability.

From Definitions 6 and 7, if $\phi^{\mathrm{T}}=\phi_{1}^{\mathrm{T}} \vee \cdots \vee \phi_{m^{\mathrm{T}}}^{\mathrm{T}}$ is structurally transferable from $\phi^{\mathrm{S}}=\phi_{1}^{\mathrm{S}} \vee \cdots \vee \phi_{m^{\mathrm{S}}}^{\mathrm{S}}$, then for all index $i \in\left[1, m^{\mathrm{T}}\right]$, the sequential conjunctive subformulas

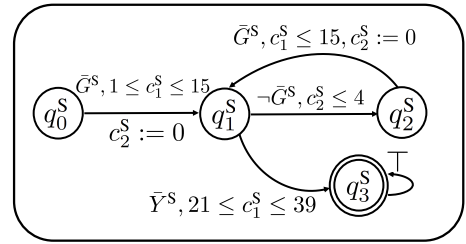

(a)

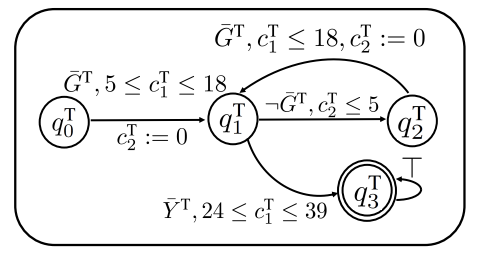

(b)

Figure 4: The deterministic timed automata (DTA) of two structurally equivalent formulas (a) $\diamond_{[1,15]} \square_{[0,4]} \bar{G}^{\mathrm{S}} \wedge \diamond_{[21,39]} \bar{Y}^{\mathrm{S}}$ and (b) $\diamond_{[5,18]} \square_{[0,5]} \bar{G}^{\mathrm{T}} \wedge \nabla_{[24,39]} \bar{Y}^{\mathrm{T}}$. The locations $q_{0}^{\mathrm{S}}, q_{1}^{\mathrm{S}}, q_{2}^{\mathrm{S}}$ and $q_{3}^{\mathrm{S}}$ correspond to $q_{0}^{\mathrm{T}}, q_{1}^{\mathrm{T}}, q_{2}^{\mathrm{T}}$ and $q_{3}^{\mathrm{T}}$, respectively. The atomic predicate $\bar{G}^{\mathrm{S}}$ and $\bar{Y}^{\mathrm{S}}$ correspond to $\bar{G}^{\mathrm{T}}$ and $\bar{Y}^{\mathrm{T}}$, respectively. The clocks $c_{1}^{\mathrm{S}}$ and $c_{2}^{\mathrm{S}}$ correspond to $c_{1}^{\mathrm{T}}$ and $c_{2}^{\mathrm{T}}$, respectively.

$\phi_{i}^{\mathrm{T}}$ and $\phi_{\hat{i}}^{\mathrm{S}}$ are structurally equivalent, where $\hat{i}=i \bmod m^{\mathrm{S}}$ (where mod denotes the modulo operation). For the DTA $\mathcal{A}^{\phi_{i}^{\mathrm{T}}}$ and $\mathcal{A}^{\phi_{i}^{\mathrm{S}}}$ constructed from $\phi_{i}^{\mathrm{T}}$ and $\phi_{\hat{i}}^{\mathrm{S}}$ respectively, it can be proven that we can establish bijective mappings: $\xi_{\Sigma}^{i, \hat{i}}$ : $2^{A P} \rightarrow 2^{A P}, \xi_{\mathcal{Q}}^{i, \hat{i}}: \mathcal{Q}^{\phi_{i}^{\mathrm{T}}} \rightarrow \mathcal{Q}^{\phi_{\hat{i}}^{\mathrm{s}}}$ and $\xi_{C}^{i, \hat{i}}: C^{\phi_{i}^{\mathrm{T}}} \rightarrow C^{\phi_{\hat{i}}^{\mathrm{s}}}$ such that the structures of $\mathcal{A}^{\phi_{i}^{\mathrm{T}}}$ and $\mathcal{A}^{\phi_{\hat{i}}^{\mathrm{S}}}$ are preserved under these bijective mappings [Glushkov, 1961]. Specifically, we have $\xi_{\mathcal{Q}}^{i, \hat{i}}\left(q^{0 \phi_{i}^{\mathrm{T}}}\right)=q^{0 \phi_{\hat{i}}^{\mathrm{S}}}, \xi_{\mathcal{Q}}^{i, \hat{i}}\left[\mathcal{F}^{\phi_{i}^{\mathrm{T}}}\right]=\mathcal{F}^{\phi_{\hat{i}}^{\mathrm{S}}}\left(\right.$ where $\xi_{\mathcal{Q}}^{i, \hat{i}}\left[\mathcal{F}^{\phi_{i}^{\mathrm{T}}}\right]$ denotes the point-wise application of $\xi_{\mathcal{Q}}^{i, \hat{i}}$ to elements of $\mathcal{F}^{\phi_{i}^{\mathrm{T}}}$. Besides, for any $\rho \in 2^{A P}$ and any $q, q^{\prime} \in \mathcal{Q}^{\phi_{i}^{\mathrm{T}}}$, we have that

$$
e^{\phi_{i}^{\mathrm{T}}}=\left(q, \rho, q^{\prime}, \varphi_{C_{1}}^{\phi_{i}^{\mathrm{T}}}, r_{C_{1}}^{\phi_{i}^{\mathrm{T}}}\right) \in \Delta^{\phi_{i}^{\mathrm{T}}}
$$

holds if and only if

$$
e^{\phi_{\hat{i}}^{\mathrm{S}}}=\left(\xi_{\mathcal{Q}}^{i, \hat{i}}(q), \xi_{\Sigma}^{i, \hat{i}}(\rho), \xi_{\mathcal{Q}}^{i, \hat{i}}\left(q^{\prime}\right), \varphi_{C_{2}}^{\phi_{\hat{i}}^{\mathrm{S}}}, r_{C_{2}}^{\phi_{\hat{i}}^{\mathrm{S}}}\right) \in \Delta^{\phi_{\hat{i}}^{\mathrm{S}}}
$$

holds, where $C_{1}=C^{\phi_{i}^{\mathrm{T}}}$ and $C_{2}=\xi_{C}^{i, \hat{i}}\left[C^{\phi_{i}^{\mathrm{T}}}\right]$. See Figure 4 for an illustrative example.

Algorithm 2 is for the transfer of the extended Q-functions. For all indices $i$, we first identify a unique primitive MITL $_{f}$ formula $\phi_{i}^{j, \mathrm{~T}}$ (as in $\phi_{i}^{\mathrm{T}}=\phi_{i}^{1, \mathrm{~T}} \wedge \cdots \wedge \phi_{i}^{n_{i}, \mathrm{~T}}$ ) that is to be satisfied at each extended state $\chi_{i}^{\mathrm{T}}=\left(s^{\mathrm{T}}, q^{\mathrm{T}}, v^{\mathrm{T}}\right)$ (Line 3). Specifically, according to $q^{\mathrm{T}}$ and $v^{\mathrm{T}}$, we identify the index $j$ such that $\phi_{i}^{1, \mathrm{~T}}, \ldots, \phi_{i}^{(j-1), \mathrm{T}}$ are already satisfied while $\phi_{i}^{j, \mathrm{~T}}$ is still not satisfied.

Next, we identify the state, location and clock valuation in the extended state $\chi_{\hat{i}}^{\mathrm{S}}$ that are the most similar to $s^{\mathrm{T}}, q^{\mathrm{T}}$ and $v^{\mathrm{T}}$ respectively in the extended state $\chi_{i}^{\mathrm{T}}$.

Identification of State: We first identify the atomic predicate $\rho_{i}^{j, \mathrm{~T}}$ in the primitive $\mathrm{MITL}_{f}$ formula $\phi_{i}^{j, \mathrm{~T}}$. Then we identify the atomic predicate $\rho^{\mathrm{S}}$ corresponding to $\rho_{i}^{j, \mathrm{~T}}$ through the 


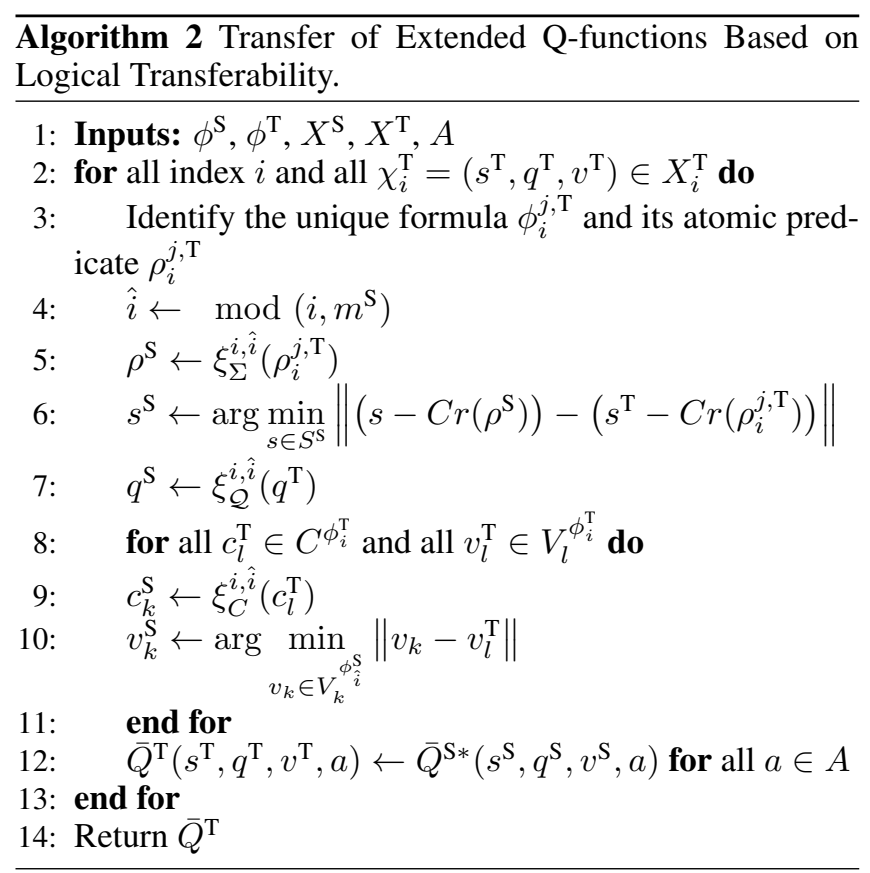

mapping $\xi_{\Sigma}^{i, \hat{i}}$ (Line 5). As in the introductory example, at the locations $q_{0}^{\mathrm{S}}$ and $q_{0}^{\mathrm{T}}$, we first identify the atomic predicate $\bar{G}^{\mathrm{T}}$, then the mapping $\xi_{\Sigma}^{1,1}$ maps $\bar{G}^{\mathrm{T}}$ to its corresponding atomic predicate $\bar{G}^{\mathrm{S}}$. We use $\operatorname{Cr}(\rho)$ to denote the centroid of the region corresponding to the atomic predicate $\rho$ and $\|\cdot\|$ to denote the 2-norm. We identify the state $s^{\mathrm{S}}$ in $S^{\mathrm{S}}$ such that the relative position of $s^{\mathrm{S}}$ with respect to $\operatorname{Cr}\left(\rho^{\mathrm{S}}\right)$ is the most similar (measured in Euclidean distance) to the relative position of $s^{\mathrm{T}}$ with respect to $\operatorname{Cr}\left(\rho_{i}^{j, \mathrm{~T}}\right)$ (Line 6).

Identification of Location: We identify the location $q^{\mathrm{S}}$ corresponding to $q^{\mathrm{T}}$ through the mapping $\xi_{\mathcal{Q}}^{i, \hat{i}}$ (Line 7). As in the introductory example, the mapping $\xi_{\mathcal{Q}}^{1,1}$ maps the locations $q_{0}^{\mathrm{T}}, q_{1}^{\mathrm{T}}, q_{2}^{\mathrm{T}}$ and $q_{3}^{\mathrm{T}}$ to the locations $q_{0}^{\mathrm{S}}, q_{1}^{\mathrm{S}}, q_{2}^{\mathrm{S}}$ and $q_{3}^{\mathrm{S}}$, respectively (see Figure 4).

Identification of Clock Valuation: For each clock $c_{l}^{\mathrm{T}} \in C^{\phi_{i}^{\mathrm{T}}}$, we identify the clock $c_{k}^{\mathrm{S}}$ corresponding to $c_{l}^{\mathrm{T}}$ through the mapping $\xi_{C}^{i, \hat{i}}$ (Line 9). As in the introductory example, the mapping $\xi_{C}^{1,1}$ maps the clocks $c_{1}^{\mathrm{T}}$ and $c_{2}^{\mathrm{T}}$ to the corresponding clocks $c_{1}^{\mathrm{S}}$ and $c_{2}^{\mathrm{S}}$, respectively (see Figure 4 ). Then for each clock valuation $v_{l}^{\mathrm{T}} \in V_{l}^{\phi_{i}^{\mathrm{T}}}$, we identify the (scalar) clock valuation $v_{k}^{\mathrm{S}} \in V_{k}^{\phi_{\hat{i}}^{\mathrm{S}}}$ which is the most similar (in scalar value) to $v_{l}^{\mathrm{T}}$ (Line 10).

In this way, $\bar{Q}^{\mathrm{S} *}\left(s^{\mathrm{S}}, q^{\mathrm{S}}, v^{\mathrm{S}}, a\right)$ from the source task are transferred to $\bar{Q}^{\mathrm{T}}\left(s^{\mathrm{T}}, q^{\mathrm{T}}, v^{\mathrm{T}}, a\right)$ in the target task. Finally, we perform Q-learning of $\mathcal{T}^{\mathrm{T}}$ in the extended state space, starting with the transferred extended Q-functions $\bar{Q}^{\mathrm{T}}\left(s^{\mathrm{T}}, q^{\mathrm{T}}, v^{\mathrm{T}}, a\right)$.

\section{Implementation}

In this section, we illustrate the proposed approach on a case study $^{2}$. We consider the introductory example in the $9 \times 9$ gridworld as shown in Figure 1. The robot has three possible actions at each time step: go straight, turn left or turn right. After going straight, the robot may slip to adjacent cells with probability of 0.04 . After turning left or turning right, the robot may stay in the original direction with probability of 0.03. We first perform Q-learning on the $\tau$-states (i.e., the $\tau$-horizon trajectory involving the current state and the most recent $\tau-1$ past states, see [Aksaray et al., 2016], we set $\tau=5$ ) for the source task and the target task. We set $\alpha=0.8$ and $\gamma=0.99$. For each episode, the initial state is randomly selected.

We use the first 10000 episodes of Q-learning as the data collection phase. From the source task, all the 46 trajectories with cumulative rewards above 0 are labeled as 1 , and 200 trajectories randomly selected out of the remaining 9954 trajectories are labeled as -1 . From the target task, all the 19 trajectories with cumulative rewards above 0 are labeled as 1 and 200 trajectories randomly selected out of the remaining 9981 trajectories are labeled as -1 .

For the inference problem (Problem 1), we set $\varrho_{\text {th }}=4$ and $\zeta=0.95$. For Algorithm 1 , we set $\lambda=0.01$ and $h_{\max }=2$. We use the position of the robot as the state, and the atomic predicates $\rho$ correspond to the rectangular regions in the $9 \times 9$ gridworld. For computing the information gain of $\mathrm{MITL}_{f}$ formulas, we use the uniform distribution for the prior probability distribution $\mathcal{G}_{L}$. Following the first two steps illustrated in Section 4.2, we obtain the following satisfying formulas:

$$
\begin{aligned}
\phi^{\mathrm{S}} & =\diamond_{[1,15]} \square_{[0,4]} \bar{G}^{\mathrm{S}} \wedge \diamond_{[21,39]} \bar{Y}^{\mathrm{S}} \text { and } \\
\phi^{\mathrm{T}} & =\diamond_{[5,18]} \square_{[0,5]} \bar{G}^{\mathrm{T}} \wedge \diamond_{[24,39]} \bar{Y}^{\mathrm{T}},
\end{aligned}
$$

where

$$
\begin{aligned}
& \bar{G}^{\mathrm{S}}=(x>=3) \wedge(x<=4) \wedge(y>=3) \wedge(y<=5), \\
& \bar{Y}^{\mathrm{S}}=(x>=7) \wedge(x<=8) \wedge(y>=5) \wedge(y<=8), \\
& \bar{G}^{\mathrm{T}}=(x>=5) \wedge(x<=6) \wedge(y>=6) \wedge(y<=7), \\
& \bar{Y}^{\mathrm{T}}=(x>=4) \wedge(x<=7) \wedge(y>=1) \wedge(y<=2) .
\end{aligned}
$$

$\phi^{\mathrm{S}}$ reads as "first reach $\bar{G}^{\mathrm{S}}$ during the time interval [1, 15] and stay there for 4 time units, then reach $\bar{Y}^{\mathrm{S}}$ during the time interval $[21,39]$ ". $\phi^{\mathrm{T}}$ reads as "first reach $\bar{G}^{\mathrm{T}}$ during the time interval $[5,18]$ and stay there for 5 time units, then reach $\bar{Y}^{\mathrm{T}}$ during the time interval $[24,39]$ ]". The regions $\bar{G}^{\mathrm{S}}, \bar{Y}^{\mathrm{S}}, \bar{G}^{\mathrm{T}}$ and $\bar{Y}^{\mathrm{T}}$ are shown in Figure 5 (a) (b). It can be seen that $\phi^{\mathrm{T}}$ is structurally equivalent with $\phi^{\mathrm{S}}$, hence logical transferability is identified.

For comparison, we also obtain $\phi^{\prime} \mathrm{T}$ without considering the information gain, i.e., by setting $\lambda=0$ in (3):

$$
\phi^{\prime \mathrm{T}}=\diamond_{[1,18]} \square_{[0,4]} \bar{G}^{\mathrm{T}} \wedge \diamond_{[24,40]} \bar{Y}^{\prime \mathrm{T}},
$$

where

$$
\begin{aligned}
& \bar{G}^{\mathrm{T}}=(x>=5) \wedge(x<=6) \wedge(y>=6) \wedge(y<=7), \\
& \bar{Y}^{\prime} \mathrm{T}=(x>=0) \wedge(x<=8) \wedge(y>=0) \wedge(y<=2) .
\end{aligned}
$$

${ }^{2}$ Dur to space limitations, an additional case study can be found in the longer version https://bit.ly/2Zjx $7 \mathrm{mt}$. 


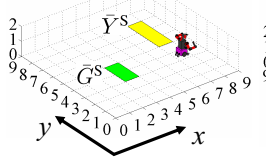

(a)

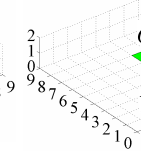

(b)

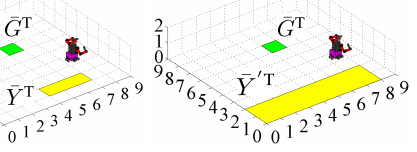

(c)
Figure 5: Inferred regions in the case study.

$\phi^{\prime} \mathrm{T}$ reads as "first reach $\bar{G}^{\mathrm{T}}$ during the time interval $[1,18]$ and stay there for 4 time units, then reach $\bar{Y}^{\prime} \mathrm{T}$ during the time interval $[24,40]$ '. $\bar{G}^{\mathrm{T}}$ and $\bar{Y}^{\prime} \mathrm{T}$ are shown in Figure 5 (c). It can be seen that $\phi^{\mathrm{T}}$ implies $\phi^{\prime}$, hence $\phi^{\prime} \mathrm{T}$ is less informative than $\phi^{\mathrm{T}}$ with respect to the prior probability distribution $\mathcal{G}_{L}$.

We use Method I to refer to the Q-learning on the $\tau$-states. In comparison with Method I, we perform Q-learning in the extended state space with the following three methods:

Method II: Q-learning with $\phi^{\prime} \mathrm{T}$ (i.e., on the extended state that includes the locations and clock valuations of the timed automata constructed from $\phi^{\prime} \mathrm{T}$ ).

Method III: Q-learning with $\phi^{\mathrm{T}}$.

Method IV: Q-learning with $\phi^{\mathrm{T}}$ and starting from the transferred extended Q-functions.

Figure 6 shows the learning results with the four different methods. Method I takes an average of 834590 episodes to converge to the optimal policy (with the first 50000 episodes shown in Figure 6), while Method III and Method IV take an average of 13850 episodes and 2220 episodes for convergence to the optimal policy, respectively. It should be noted that although Method II performs better than Method I in the first 50000 episodes, it does not achieve optimal performance in 2 million episodes (as $\phi^{\prime} \mathrm{T}$ is not sufficiently informative). In sum, the sampling efficiency for the target task is improved by up to one order of magnitude by performing RL in the extended state space with the inferred formula $\phi^{\mathrm{T}}$, and further improved by up to another order of magnitude using the transferred extended Q-functions.

\section{Discussions}

We proposed a transfer learning approach for temporal tasks based on logical transferability. We have shown the improvement of sampling efficiency in the target task using the pro-

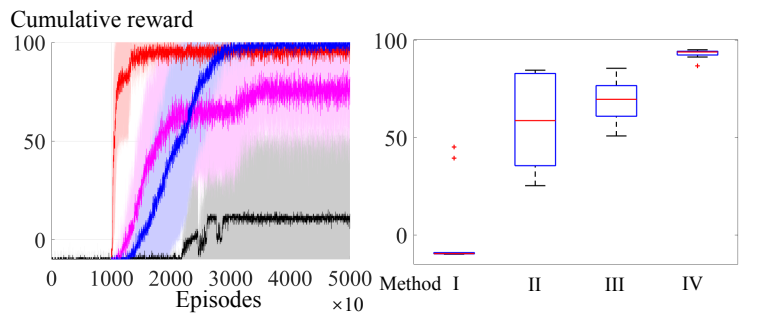

Figure 6: Learning results in the case study: cumulative rewards of 10 independent simulation runs averaged for every 10 episodes (left) and boxplot of the 10 runs for the average cumulative rewards of 40000 episodes after the data collection phase (right). Black: Method I; magenta: Method II; blue: Method III; red: Method IV. posed method.

There are several limitations of the current approach, which leads to possible directions for future work. Firstly, the proposed logical transferability is a qualitative measure of the logical similarities between the source task and the target task. Quantitative measures of logical similarities can be further established using similarity metrics between the inferred temporal logic formulas from the two tasks. Secondly, as some information about the task may not be discovered during the initial episodes of reinforcement learning (especially for more complicated tasks), the inferred temporal logic formulas can be incomplete or biased. We will develop methods for more complicated tasks by either breaking the tasks into simpler subtasks, or iteratively performing inference of temporal logic formulas and reinforcement learning as a closed loop process. Finally, we use Q-learning as the underlying learning algorithm for the transfer learning approach. The same methodology can be also applied to other forms of reinforcement learning, such as actor-critic methods or modelbased reinforcement learning.

\section{Acknowledgements}

This research was partially supported by AFOSR FA955019-1-0005, DARPA D19AP00004, NSF 1652113 and ONR N00014-18-1-2829.

\section{References}

[Aksaray et al., 2016] Derya Aksaray, Austin Jones, Zhaodan Kong, Mac Schwager, and Calin Belta. Q-learning for robust satisfaction of signal temporal logic specifications. In IEEE CDC'16, pages 6565-6570, Dec 2016.

[Alshiekh et al., 2018] Mohammed Alshiekh, Roderick Bloem, Rüdiger Ehlers, Bettina Könighofer, Scott Niekum, and Ufuk Topcu. Safe reinforcement learning via shielding. In $A A A I^{\prime} 18,2018$.

[Alur and Dill, 1994] Rajeev Alur and David L. Dill. A theory of timed automata. Theoretical Computer Science, 126:183-235, 1994.

[Alur et al., 1996] Rajeev Alur, Tomás Feder, and Thomas A. Henzinger. The benefits of relaxing punctuality. J. ACM, 43(1):116-146, January 1996.

[Bombara et al., 2016] Giuseppe Bombara, Cristian-Ioan Vasile, Francisco Penedo, Hirotoshi Yasuoka, and Calin Belta. A decision tree approach to data classification using signal temporal logic. In Proc. HSCC'16, pages 1-10, 2016.

[Eberhart and Shi, 2001] Eberhart and Yuhui Shi. Particle swarm optimization: developments, applications and resources. In Proceedings of the 2001 Congress on Evolutionary Computation (IEEE Cat. No.01TH8546), volume 1, pages 81-86 vol. 1, May 2001.

[Fu and Topcu, 2014] Jie Fu and Ufuk Topcu. Probably approximately correct MDP learning and control with temporal logic constraints. Robotics: Science and Systems, abs/1404.7073, 2014. 
[Glushkov, 1961] Victor M. Glushkov. The abstract theory of automata. Russian Mathematical Surveys, 16(5):1, 1961.

[Gruber et al., 2005] Hermann Gruber, Markus Holzer, Astrid Kiehn, and Barbara König. On timed automata with discrete time - structural and language theoretical characterization. In Developments in Language Theory, pages 272-283. Springer Berlin Heidelberg, 2005.

[Hoxha et al., 2017] Bardh Hoxha, Adel Dokhanchi, and Georgios Fainekos. Mining parametric temporal logic properties in model-based design for cyber-physical systems. International Journal on Software Tools for Technology Transfer, pages 79-93, Feb 2017.

[Kong et al., 2017] Zhaodan Kong, Austin Jones, and Calin Belta. Temporal logics for learning and detection of anomalous behavior. IEEE TAC, 62(3):1210-1222, Mar. 2017.

[Kress-Gazit et al., 2011] Hadas Kress-Gazit, Tichakorn Wongpiromsarn, and Ufuk Topcu. Correct, reactive, high-level robot control. IEEE Robotics Automation Magazine, 18(3):65-74, Sept 2011.

[Li et al., 2017] Xiao Li, Cristian-Ioan Vasile, and Calin Belta. Reinforcement learning with temporal logic rewards. In Proc. IROS'17, pages 3834-3839, Sept 2017.

[Mihalkova et al., 2007] Lilyana Mihalkova, Tuyen N. Huynh, and Raymond J. Mooney. Mapping and revising markov logic networks for transfer learning. In Proc. AAAI'07, pages 608-614, Vancouver, BC, July 2007.

[Neider and Gavran, 2018] Daniel Neider and Ivan Gavran. Learning linear temporal properties. In Formal Methods in Computer Aided Design (FMCAD), pages 1-10, 2018.

[Ouaknine and Worrell, 2005] Joël Ouaknine and James Worrell. On the decidability of metric temporal logic. In Proc. Annual IEEE Symposium on Logic in Computer Science, LICS'05, pages 188-197, Washington, DC, USA, 2005. IEEE Computer Society.

[Pnueli, 1977] Amir Pnueli. The temporal logic of programs. In Proc. 18th Annu. Symp. Found. Computer Sci., pages 46-57, Washington, D.C., USA, 1977.

[Shah et al., 2018] Ankit Shah, Pritish Kamath, Julie A Shah, and Shen Li. Bayesian inference of temporal task specifications from demonstrations. In S. Bengio, H. Wallach, H. Larochelle, K. Grauman, N. Cesa-Bianchi, and R. Garnett, editors, NeurIPS, pages 3808-3817. Curran Associates, Inc., 2018.

[Taylor and Stone, 2007] Matthew E. Taylor and Peter Stone. Cross-domain transfer for reinforcement learning. In Proc. ICML'07, pages 879-886, New York, NY, USA, 2007. ACM.

[To et al., 2016] Son Thanh To, Mark Roberts, homas Apker, Benjamin Johnson, and David W. Aha. Mixed propositional metric temporal logic: A new formalism for temporal planning. In D. Magazzeni, S. Sanner, \& S. Thiebaux (Eds.) Planning for Hybrid Systems: Papers from the AAAI Workshop (Technical Report WS-16-13), Phoenix, AZ, 2016. AAAI Press.
[Toro Icarte et al., 2018a] Rodrigo Toro Icarte, Toryn Q. Klassen, Richard Valenzano, and Sheila A. McIlraith. Advice-based exploration in model-based reinforcement learning. In Proc. CCAI'18, pages 72-83, 2018.

[Toro Icarte et al., 2018b] Rodrigo Toro Icarte, Toryn Q. Klassen, Richard Valenzano, and Sheila A. McIlraith. Teaching multiple tasks to an RL agent using LTL. In $A A$ $M A S^{\prime} 18$, pages 452-461, Richland, SC, 2018.

[Torrey and Shavlik, 2010] Lisa Torrey and Jude Shavlik. Policy transfer via markov logic networks. In Luc De Raedt, editor, Inductive Logic Programming, pages 234-248, Berlin, Heidelberg, 2010. Springer Berlin Heidelberg.

[Torrey et al., 2008] Lisa Torrey, Jude W. Shavlik, Trevor Walker, and Richard Maclin. Rule extraction for transfer learning. In Rule Extraction from Support Vector Machines, pages 67-82, 2008.

[Vazquez-Chanlatte et al., 2018] Marcell VazquezChanlatte, Susmit Jha, Ashish Tiwari, Mark K. Ho, and Sanjit A. Seshia. Learning task specifications from demonstrations. In NeurIPS, pages 5372-5382, 2018.

[Wang and Taylor, 2017] Zhaodong Wang and Matthew E. Taylor. Improving reinforcement learning with confidence-based demonstrations. In Proc. IJCAI'17, pages 3027-3033. AAAI Press, 2017.

[Watkins and Dayan, 1992] Christopher J. C. H. Watkins and Peter Dayan. Q-learning. Machine Learning, 8(3):279292, May 1992.

[Wen et al., 2017] Min Wen, Ivan Papusha, and Ufuk Topcu. Learning from demonstrations with high-level side information. In Proc. IJCAI'17, pages 3055-3061, 2017.

[Xu and Julius, 2018] Zhe Xu and Agung Julius. Census signal temporal logic inference for multiagent group behavior analysis. IEEE Trans. Autom. Sci. Eng., 15(1):264-277, Jan. 2018.

[Xu et al., 2018] Zhe Xu, Sayan Saha, Botao Hu, Sandipan Mishra, and Agung Julius. Advisory temporal logic inference and controller design for semiautonomous robots. IEEE Trans. Autom. Sci. Eng., pages 1-19, 2018.

[Xu et al., 2019] Zhe Xu, Melkior Ornik, Agung Julius, and Ufuk Topcu. Information-guided temporal logic inference with prior knowledge. In Proc. IEEE Amer. Control Conf., 2019.

[Zhuo and Yang, 2014] Hankz Hankui Zhuo and Qiang Yang. Action-model acquisition for planning via transfer learning. Artificial Intelligence, 212:80 - 103, 2014. 